\title{
The Non-linear Health Consequences of Living in Larger Cities
}

\author{
Luis E C Rocha, Anna E. Thorson, and Renaud Lambiotte
}

\begin{abstract}
Urbanization promotes economy, mobility, access, and availability of resources, but on the other hand, generates higher levels of pollution, violence, crime, and mental distress. The health consequences of the agglomeration of people living close together are not fully understood. Particularly, it remains unclear how variations in the population size across cities impact the health of the population. We analyze the deviations from linearity of the scaling of several health-related quantities, such as the incidence and mortality of diseases, external causes of death, wellbeing, and health care availability, in respect to the population size of cities in Brazil, Sweden, and the USA. We find that deaths by non-communicable diseases tend to be relatively less common in larger cities, whereas the per capita incidence of infectious diseases is relatively larger for increasing population size. Healthier lifestyle and availability of medical support are disproportionally higher in larger cities. The results are connected with the optimization of human and physical resources and with the non-linear effects of social networks in larger populations. An urban advantage in terms of health is not evident, and using rates as indicators to compare cities with different population sizes may be insufficient.
\end{abstract}

KEYWORDS Urbanization, Population dynamics, Public health, Urban health, Complex systems

\section{INTRODUCTION}

Migration from rural to urban areas reached a shift point in 2007 when the United Nations estimated that the world urban population had surpassed the rural. ${ }^{1}$ This is a complicated phenomenon motivated by economic reasons such as employment opportunities and higher salaries, and also a consequence of improved agricultural technology that demands less human resources on the field. Migration between smaller and larger cities has contributed to urbanization, and long-term projections estimate that cities will keep increasing in population size. ${ }^{2}$ The aggregation of people in larger cities has positive and negative consequences for the society, ${ }^{3-5}$ and the so-called urban advantage ${ }^{6}$ is not yet a consensus. While urbanization promotes innovation, develops the local economy, ${ }^{7,8}$ and optimizes resources, ${ }^{8}$ as for example the number and quality of specialized hospitals, it is also associated to higher criminal activity, ${ }^{9}$ homicides, ${ }^{10}$ carbon emissions, ${ }^{11-13}$ and mental distress. ${ }^{14}$ These and other fundamental amenities of urban life, such as efficient transportation systems or sanitation and wastewater management, all combine to the pool of

Rocha and Lambiotte are with the Department of Mathematics and naXys, Université de Namur, Namur, Belgium; Rocha and Thorson are with the Department of Public Health Sciences, Karolinska Institutet, Stockholm, Sweden.

Correspondence: Luis E C Rocha, Department of Public Health Sciences, Karolinska Institutet, Stockholm, Sweden. (E-mail: luis.rocha@ki.se) 
factors affecting health and wellbeing. ${ }^{15,16}$ Efforts have been made to map the complex interconnections between all these factors aiming to develop the concept of healthy cities. ${ }^{15}$ Complexity, however, means that the behavior of the system is not simply the sum of the behavior of its individual parts. Complex systems, like cities, may incorporate, among other characteristics, highly heterogeneous multi-level networks, fitness, feedback, and chaotic behavior. ${ }^{17,18}$ All combined, these characteristics may generate non-linear responses to linear inputs.

Health-related quantities are characterized by a number of statistical indicators to summarize features of a given population aiming to rank and compare individual cities. These indicators are widely used by policy makers to identify areas that need further attention or to quantify and monitor achievements of previous efforts. Wellknown indicators include life expectancy, death and prevalence rates, income, coverage, and so on. Many times, a city-level indicator such as a rate is defined by dividing a quantity of interest by the population size of the respective city, sometimes presented as the number of cases per number of individuals, e.g., 100,000 or 1 (per capita), within a given time interval. The procedure to define a rate implicitly assumes a linear relation between the dependent and the independent variables. ${ }^{9}$ Technically, this linear assumption allows an unbiased per capita analysis only if the quantity under observation is size-independent. On the other hand, the non-linearity, represented by the parameter $\alpha$ in Eq. (1), means that cities with larger populations $P$ observe a relative per capita increase $(\alpha>1)$ or decrease $(\alpha<1)$ of the variable $Q$. The parameter $\beta$ is a constant and does not affect the linear scaling.

$$
Q=\beta P^{\alpha}
$$

The scaling parameter $\alpha$ thus indicates the relative advantage of living in larger cities by capturing the deviations from the linear scaling. The use of Eq. (1) is motivated by the study of power-law scaling between characteristics of living creatures and their body size. Allometry dates back to $1936,{ }^{19}$ but more recently, it has been adapted to study the relation between characteristics of cities and their population through a metaphor between cities and living creatures. ${ }^{8}$ While some theories have been proposed to justify power-law relations in biological systems, ${ }^{20}$ a robust theory of scaling for cities is still missing. The estimation of these scaling relations contributes to understand the macroscopic impact of the population size of cities in health, ${ }^{21}$ particularly to rank cities across a country. It also reflects the consequences of the increasing complexity of daily interactions between people and the available resources observed in cities with larger populations that eventually affect health variables. ${ }^{22}$

In this paper, we analyze the scaling laws of several health-related variables in Brazil, Sweden, and the USA. These countries have different income levels, wealth distribution, cultural background, and health care and governmental policies. The lack of standardized data for multiple countries restricts the generalizability of the results; thus, caution should guide causality associations between the scaling of the different variables particularly because some results are country-specific. Nevertheless, whenever possible, we make a comparative analysis between the different contexts. We particularly look at the prevalence of several infectious and noncommunicable diseases, causes of death, children-related health and social variables, wellbeing, and availability and access to health care facilities. We emphasize however that results are not necessarily universal but country-specific. 


\section{METHODS}

\section{Datasets}

For each country, we collect city-level data on the variable of interest and on the population size. To avoid misinterpretation of the concept of city, we simply use the finest resolution of urban area as available in the respective datasets. This translates to nearly 5550 places in Brazil, 210 in Sweden, and 980 in the USA. Note that the exact number of cities (i.e., data points) depends on the particular study variable since cities with no reported cases are removed for the statistics. There is also no standardization across countries, and we use the definition of the variables as they are described on each dataset. We consider the place of residence and not the local of occurrence for the statistics. The Brazilian dataset is obtained from "Data SUS" that is the department of informatics of the Brazilian publicly funded health care system (www2.datasus.gov.br). The Swedish dataset comes from "Folkhälsomyndigheten," the public health agency of Sweden (www.folkhalsomyndigheten.se). The datasets related to the USA are obtained from "The County Health Rankings \& Roadmaps program," a consortium between the Robert Wood Johnson Foundation and the University of Wisconsin Population Health Institute (www.countyhealthrankings.org).

\section{Analysis}

We initially construct two vectors, $Q$ and $P$, which entries $q_{\mathrm{i}}$ and $p_{\mathrm{i}}$ contain, respectively, the variable of interest and the population size of the city, $i$. To estimate the coefficients $\alpha$ and $\beta$, we first take the logarithm on both sides of Eq. (1).

$$
\log (Q)=\log (\beta)+\alpha \log (P)
$$

to linearize the original non-linear formula such that $A=\log (\beta)$ and $B=\alpha$ in $y=A+$ $B x$. Typically, the range of values of the population sizes spans several orders of magnitude. The logarithm operator reduces the difference in magnitude of these values and allows the estimation of the parameters using a simple regression analysis on Eq. (2). Alternatively, one may apply more advanced methods, e.g., weighted least squares, directly on Eq. (1). ${ }^{9}$ We fit the data points in the intervals [50,0005,000,000] (for Brazil and the USA) and [10,000-500,000] (for Sweden) of population size to remove the small cities that typically cause a large dispersion for low values and to remove very large cities that are statistically underrepresented. If no cases are observed in a city for a certain variable, the city is not used for the statistics. This procedure is standard in the literature. ${ }^{8,23}$ The exponents $\alpha$ and $\beta$, the respective confidence intervals, and the adjusted correlations coefficients $R^{2}$ are estimated using the statistical software R (www.r-project.org).

\section{Super- vs. Sub-linear Scaling}

The non-linear dependence between variables is not as intuitive as the linear relation. Mathematically, non-linearity is characterized by either the so-called superlinear $(\alpha>1)$ or the sub-linear $(\alpha<1)$ scaling exponent that measures the deviation from the linear behavior $(\alpha=1)$ in Eq. (1). Note that the parameter $\beta$ that is a constant measures the intensity of the relation between the variables and is not relevant to determine the non-linearity. Using Eq. (1), one can show that the relative increase in a given outcome $Q$ for an increase $P_{2}=\delta P_{1}$ in the population is $\left(Q_{2}-Q_{1}\right) /$ $Q_{1}=\delta^{\alpha}-1$, where $\delta$ is a constant number and $Q_{1}$ and $Q_{2}$ correspond to the value of 
the variable $Q$ (e.g., number of cases, counts) associated respectively to the population size $P_{1}$ and $P_{2}$. Therefore, if the dependence between the two variables is linear, $\alpha=1$ and $Q_{2}=\delta Q_{1}$. On the other hand, in case of non-linear scaling, i.e., $\alpha \neq$ 1 , the relative increase becomes non-trivial. To understand quantitatively this difference, Fig. 1a shows the relative quantity $\left(Q_{2}-Q_{1}\right) / Q_{1}$ for a given change $\delta$ in the population size, for different values of the exponent $\alpha$. To facilitate the analysis, we arbitrarily define ranges for the deviations to the linear behavior based on typical results reported in the literature: $0.95 \leq \alpha \leq 1.05$ (weak deviation), $0.85 \leq \alpha<0.95$ and $1.05<\alpha \leq 1.15$ (medium), and $\alpha<0.85$ and $\alpha>1.15$ (strong). As an example, if $\alpha=1.1$ and the population increases by $100 \%$ (i.e., $\delta=2$ ), the outcome increases by $114.35 \%$. On the other hand, for $\alpha=0.9$, the same $100 \%$ increase in the population causes an increase of only $86.61 \%$ in the outcome Q. In this particular case, the nearly $15 \%$ difference accounts for a mismatch (in respect to the linear assumption) of 1500 in 10,000 counts. This example also illustrates the non-symmetry of the exponent around its linear value, i.e., the relative increase given by $\alpha=1.1$ is not equivalent to the relative decrease given by $\alpha=0.9$.

\section{RESULTS}

To illustrate a typical scale relation between a variable and the population size of a city, we show a scatter plot of the population size of cities and the number of deaths by heart attack (Fig. 1b) and the same relation for the number of abortions (Fig. 1c) in the context of Sweden. The deviation to the linear trend is not visually evident, but the number of heart attacks scales strongly sub-linearly $(\alpha=0.80$ with $95 \%$ C.I. $[0.74,0.86])$ whereas the number of abortions scales weakly super-linearly $(\alpha=1.05$ with $95 \%$ C.I. $[1.01,1.09])$ with the population size.

For infectious diseases, the exponent $\alpha$ is typically larger than one (Table 1), being strongly super-linear for sexually transmitted infections, ${ }^{24}$ meningitis, and the 2009 pandemic influenza in Brazil. Infectious diseases spread mainly through contacts between an infected host and a healthy person. ${ }^{25}$ At the city level, the high connectivity of the human contact networks, which scales super-linearly with city size ${ }^{26}$ facilitates the spread of infections and may be the responsible for these strong deviations. The fact that the 2010 pandemic influenza scales linearly with city population possibly indicates an effective response after the epidemic outbreak in the previous year. It has been reported that in the influenza pandemic of 1918, the
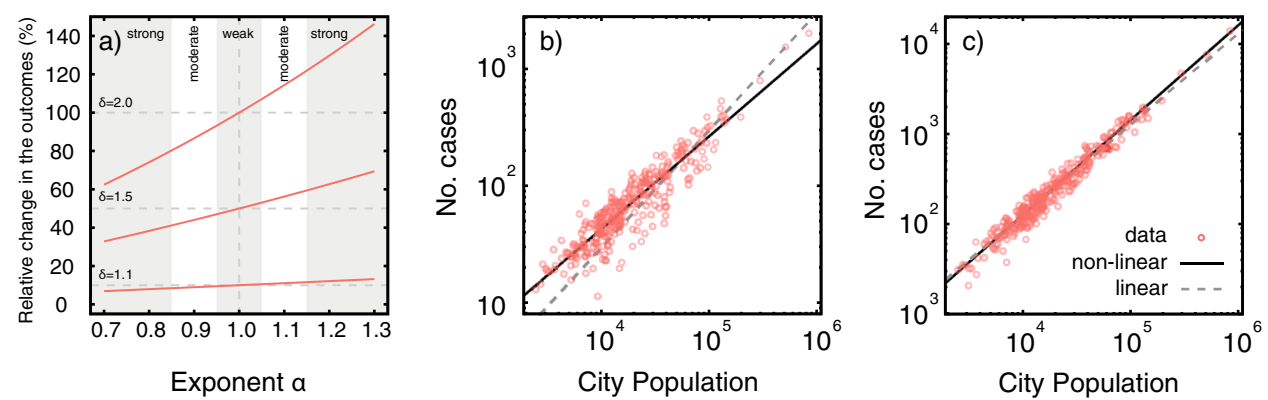

FIG. 1 a Relative change in the outcome $Q,\left(Q_{2}-Q_{1}\right) / Q_{1}$ ( $y$-axis), for different values of the exponent $\alpha$ (x-axis). For $\alpha=1$, we recover the usual linear relation between population size and the outcome variable. The relation between the population size ( $x$-axis) and $\mathbf{b}$ the number of deaths by heart attack or the c number of abortions ( $y$-axis). Both axes are in log-scale. 
number of deaths scales sub-linearly with the population size of US cities. On the other hand, the same authors report that pneumonia deaths follow a linear relation. ${ }^{27}$ The sub-linear scaling of leprosy cases indicates that smaller cities are relatively more affected than larger cities. This may be related to the fact that leprosy, although with known cure, is a silent, sometimes difficult to diagnose disease, ${ }^{28}$ and therefore disproportionally affects areas with insufficient medical resources. Dengue, on the other hand, spreads through an infected mosquito that is typically prevalent in areas lacking proper sanitation and mosquito control. Although urbanized areas (e.g., slums) are associated to increased risk of infection, ${ }^{29}$ our analysis suggests that in Brazil, the risk increases linearly with the population size.

We have also identified that the number of deaths by external causes and by noncommunicable diseases typically deviates from the linear behavior (Table 1). The results indicate that larger cities have relatively less deaths by external causes such as car accidents, injury, or suicides. ${ }^{23}$ Deaths by non-external causes (noncommunicable diseases) depend on the nature of the disease and on the country. These results possibly reflect not only the local behavior (e.g., healthier lifestyle) but also an equally distributed and accessible health care system. For example, diabetes deaths and heart attacks are both strongly sub-linear in Sweden but are respectively weakly sub-linear and weakly super-linear in Brazil in 2012. In comparison to previous years, however, both indicators have decreased in Brazil. Lung cancer and chronic respiratory insufficiency are also sub-linear in Sweden but consistently super-linear in Brazil. The population size of cities may also affect children and families. We observe a relative decrease in mortality for both infants and children in larger cities, but child poverty varies from weakly sub-linear in the USA to weakly super-linear in Sweden (Table 1). According to Swedish data, there is a small tendency to find medium-size families in larger cities, where pregnant women are also more likely to perform an abortion.

Our results suggest that people have a tendency to healthier lifestyle in larger cities in the USA; for example, we observe substantially less smoking and physical inactivity and more access to healthy food (Table 2). Similarly, obesity is also proportionally less common in larger cities in both Sweden and the USA. In contrast, limited data ${ }^{30}$ suggest that smoking is disproportionally higher in larger capital cities of Brazil ( $\alpha=1.05$ with $95 \%$ C.I. [0.95, 1.15]; $\beta=6.68$ with $95 \%$ C.I. $[2.68,10.68]$ ). Such activities have a strong social component. Recent studies suggest that the social networks affect obesity ${ }^{31,32}$ and smoking habits, ${ }^{33}$ not only by peer fitness (or peer attraction) but also by influencing one's close contacts, as for example close relatives. On the other hand, citizens of larger cities are more exposed to violent crimes and have reported a disproportionally higher number of rapes (Brazil and Sweden) and household problems, including domestic violence (Brazil). In some contexts, city life also causes distress on the population reducing the psychological wellbeing (Sweden) and increasing excessive drinking (USA).

Larger cities are known to disproportionally generate knowledge and capital, to optimize resources, ${ }^{8}$ and to boost productivity due to higher social interactions. ${ }^{34,35}$ The optimization of resources also implies that cities are expected to concentrate highquality specialized health care centers and professionals. For various diseases, however, generalist clinics and physicians are sufficient to provide appropriate primary care. Health care providers (primary care, dentists, mental health, and nutritionists) are more abundant per capita in larger cities both in the USA and in Brazil (Table 2). If we compare the scaling for the case of all (specialized and primary care) physicians and only primary care physicians in Brazil, we see that larger cities concentrate a 
TABLE 1 Scaling exponent $\alpha$ and constant $\beta$ for the prevalence of infectious diseases, number of deaths by external and non-external (non-communicable diseases) causes, and childrenrelated health and social variables

\begin{tabular}{|c|c|c|c|c|c|c|c|}
\hline Indicator & Country & Period & $\alpha$ & 95 \% C.I. & $\beta$ & $95 \%$ C.I. & Adj. $R^{2}$ \\
\hline \multicolumn{8}{|c|}{ Incidence of infectious diseases } \\
\hline HIV & BRA & 1990 & 1.20 & {$[1.07,1.34]$} & 0 & {$[-4.41,4.41]$} & 0.52 \\
\hline HIV & BRA & 2012 & 1.38 & {$[1.31,1.45]$} & 0 & {$[-3.00,3.00]$} & 0.71 \\
\hline HIV & USA & $2000 \mathrm{~s}$ & 1.44 & {$[1.39,1.50]$} & 0 & {$[-2.69,2.69]$} & 0.76 \\
\hline Chlamydia & USA & 2011 & 1.14 & {$[1.11,1.18]$} & 0 & {$[-2.44,2.44]$} & 0.80 \\
\hline $\begin{array}{l}\text { Pandemic } \\
\text { influenza }\end{array}$ & BRA & 2009 & 1.20 & {$[1.04,1.37]$} & 0 & {$[-5.28,5.28]$} & 0.29 \\
\hline $\begin{array}{l}\text { Pandemic } \\
\text { influenza }\end{array}$ & BRA & 2010 & 1.00 & {$[0.89,1.11]$} & 0 & {$[-3.88,3.88]$} & 0.41 \\
\hline Meningitis & BRA & 2001 & 1.25 & {$[1.16,1.34]$} & 0 & {$[-3.44,3.44]$} & 0.63 \\
\hline Meningitis & BRA & 2012 & 1.39 & {$[1.26,1.52]$} & 0 & {$[-4.28,4.28]$} & 0.51 \\
\hline Hepatite A & BRA & 2007 & 1.08 & {$[0.93,1.23]$} & 0 & {$[-4.81,4.81]$} & 0.36 \\
\hline Hepatite A & BRA & 2012 & 1.06 & {$[0.93,1.19]$} & 0 & {$[-4.41,4.41]$} & 0.37 \\
\hline Hepatite B & BRA & 2007 & 0.98 & {$[0.86,1.09]$} & 0 & {$[-3.93,3.93]$} & 0.38 \\
\hline Hepatite B & BRA & 2012 & 1.06 & {$[0.95,1.17]$} & 0 & {$[-3.76,3.76]$} & 0.43 \\
\hline Hepatite C & BRA & 2007 & 1.08 & {$[0.93,1.23]$} & 0 & {$[-4.81,4.81]$} & 0.36 \\
\hline Hepatite C & BRA & 2012 & 1.06 & {$[0.93,1.19]$} & 0 & {$[-4.41,4.41]$} & 0.37 \\
\hline Dengue & BRA & 2001 & 0.98 & {$[0.70,1.25]$} & 0 & {$[-9.92,9.92]$} & 0.09 \\
\hline Dengue & BRA & 2012 & 1.03 & {$[0.82,1.24]$} & 0 & {$[-6.95,6.95]$} & 0.14 \\
\hline Leprosy & BRA & 2001 & 0.89 & {$[0.75,1.04]$} & 0 & {$[-4.60,4.60]$} & 0.23 \\
\hline Leprosy & BRA & 2012 & 0.77 & {$[0.64,0.90]$} & 0 & {$[-4.35,4.35]$} & 0.18 \\
\hline \multicolumn{8}{|c|}{ External causes of death } \\
\hline Car accidents & BRA & 1981 & 1.13 & {$[1.03,1.22]$} & 0 & {$[-3.47,3.47]$} & 0.60 \\
\hline Car accidents & BRA & 2012 & 0.89 & {$[0.84,0.94]$} & 0 & {$[-2.59,2.59]$} & 0.70 \\
\hline $\begin{array}{l}\text { Alcohol imp } \\
\text { driving }\end{array}$ & USA & $\begin{array}{l}2008- \\
2012\end{array}$ & 0.71 & {$[0.67,0.74]$} & 0 & {$[-2.45,2.46]$} & 0.59 \\
\hline Injury & USA & $\begin{array}{l}2006- \\
2010\end{array}$ & 0.89 & {$[0.87,0.91]$} & 0.01 & {$[-2.19,2.21]$} & 0.90 \\
\hline Suicides & BRA & 1981 & 0.84 & {$[0.74,0.95]$} & 0 & {$[-3.68,3.68]$} & 0.46 \\
\hline Suicides & BRA & 1995 & 0.92 & {$[0.84,1.01]$} & 0 & {$[-3.32,3.33]$} & 0.50 \\
\hline Suicides $65+$ & SWE & $\begin{array}{l}2008- \\
2012\end{array}$ & 0.86 & {$[0.79,1.00]$} & 0 & {$[-3.37,3.37]$} & 0.59 \\
\hline Suicides $65-$ & SWE & $\begin{array}{l}2008- \\
2012\end{array}$ & 0.95 & {$[0.87,1.03]$} & 0 & {$[-2.94,2.95]$} & 0.73 \\
\hline \multicolumn{8}{|c|}{ Non-external causes of death } \\
\hline Diabetes & BRA & 1996 & 1.22 & {$[1.13,1.31]$} & 0 & {$[-3.30,3.30]$} & 0.61 \\
\hline Diabetes & BRA & 2012 & 0.97 & {$[0.92,1.02]$} & 0 & {$[-2.65,2.65]$} & 0.70 \\
\hline Diabetes & SWE & $\begin{array}{r}2008- \\
2012\end{array}$ & 0.77 & {$[0.68,0.86]$} & 0 & {$[-3.18,3.19]$} & 0.56 \\
\hline Heart attack & BRA & 1981 & 1.25 & {$[1.12,1.38]$} & 0 & {$[-4.29,4.29]$} & 0.58 \\
\hline Heart attack & BRA & 2012 & 1.04 & {$[0.98,1.09]$} & 0 & {$[-2.71,2.71]$} & 0.70 \\
\hline Heart attack & SWE & $\begin{array}{l}2008- \\
2012\end{array}$ & 0.80 & {$[0.74,0.86]$} & 0.03 & {$[-2.66,2.71]$} & 0.76 \\
\hline $\begin{array}{l}\text { Cerebrovascular } \\
\text { accident }\end{array}$ & BRA & 1996 & 1.16 & {$[1.08,1.25]$} & 0 & {$[-3.24,3.24]$} & 0.60 \\
\hline $\begin{array}{l}\text { Cerebrovascular } \\
\text { accident }\end{array}$ & BRA & 2012 & 1.00 & {$[0.97,1.04]$} & 0 & {$[-2.44,2.44]$} & 0.83 \\
\hline
\end{tabular}


TABLE 1 Continued

\begin{tabular}{|c|c|c|c|c|c|c|c|}
\hline Indicator & Country & Period & $\alpha$ & $95 \%$ C.I. & $\beta$ & $95 \%$ C.I. & Adj. $R^{2}$ \\
\hline Lung cancer & BRA & 1981 & 1.14 & {$[1.02,1.25]$} & 0 & {$[-3.86,3.86]$} & 0.57 \\
\hline Lung cancer & BRA & 2012 & 1.16 & {$[1.09,1.22]$} & 0 & {$[-2.95,2.96]$} & 0.65 \\
\hline Lung cancer & SWE & $\begin{array}{l}2008- \\
2012\end{array}$ & 0.94 & {$[0.90,0.99]$} & 0 & {$[-2.49,2.49]$} & 0.89 \\
\hline Chronic resp insuf. & BRA & 1981 & 1.14 & {$[1.02,1.25]$} & 0 & {$[-3.90,3.90]$} & 0.53 \\
\hline Chronic resp insuf. & BRA & 2012 & 1.07 & {$[1.00,1.14]$} & 0 & {$[-2.99,2.99]$} & 0.59 \\
\hline Chronic resp insuf. & SWE & $\begin{array}{l}2008- \\
2012\end{array}$ & 0.90 & {$[0.85,0.95]$} & 0 & {$[-2.56,2.56]$} & 0.86 \\
\hline Colon cancer & BRA & 1981 & 0.77 & {$[0.66,0.88]$} & 0 & {$[-3.78,3.78]$} & 0.46 \\
\hline Colon cancer & BRA & 2012 & 1.00 & {$[0.94,1.07]$} & 0 & {$[-2.88,2.88]$} & 0.64 \\
\hline \multicolumn{8}{|c|}{ Infant- and children-related quantities } \\
\hline $\begin{array}{l}\text { Infant mortality } \\
(0-1 \text { year })\end{array}$ & BRA & 1981 & 1.10 & {$[0.98,1.22]$} & 0 & {$[-3.99,3.99]$} & 0.48 \\
\hline $\begin{array}{l}\text { Infant mortality } \\
(0-1 \text { year })\end{array}$ & BRA & 2012 & 0.98 & {$[0.94,1.03]$} & 0 & {$[-2.53,2.53]$} & 0.77 \\
\hline $\begin{array}{l}\text { Infant mortality } \\
(0-1 \text { year })\end{array}$ & USA & $2000 \mathrm{~s}$ & 0.88 & {$[0.87,0.90]$} & 0.04 & {$[-2.15,2.24]$} & 0.90 \\
\hline $\begin{array}{l}\text { Child mortality } \\
\text { (1-9 years) }\end{array}$ & BRA & 1981 & 1.02 & {$[0.91,1.12]$} & 0 & {$[-3.65,3.65]$} & 0.51 \\
\hline $\begin{array}{l}\text { Child mortality } \\
\text { (1-9 years) }\end{array}$ & BRA & 2012 & 0.94 & {$[0.88,1.00]$} & 0 & {$[-2.84,2.84]$} & 0.60 \\
\hline $\begin{array}{l}\text { Child mortality } \\
\text { (1-9 years) }\end{array}$ & USA & $2000 \mathrm{~s}$ & 0.98 & {$[0.95,1.00]$} & 0 & {$[-2.26,2.26]$} & 0.88 \\
\hline $\begin{array}{l}\text { Child poverty } \\
\left(18^{-}\right)\end{array}$ & USA & $2000 \mathrm{~s}$ & 0.97 & {$[0.94,1.00]$} & 0.07 & {$[-2.28,2.42]$} & 0.81 \\
\hline Child poverty & SWE & 2012 & 1.03 & {$[1.01,1.05]$} & 0.15 & {$[-2.05,2.34]$} & 0.98 \\
\hline Abortion & SWE & $\begin{array}{l}2007- \\
2011\end{array}$ & 1.05 & {$[1.01,1.09]$} & 0 & {$[-2.38,2.38]$} & 0.94 \\
\hline Family 0 children & SWE & 2013 & 0.99 & {$[0.97,1.02]$} & 0.42 & {$[-1.79,2.62]$} & 0.97 \\
\hline $\begin{array}{l}\text { Family } 1 \text { or } 2 \\
\text { children }\end{array}$ & SWE & 2013 & 1.05 & {$[1.03,1.07]$} & 0.06 & {$[-2.12,2.23]$} & 0.98 \\
\hline $\begin{array}{l}\text { Family 3+ } \\
\text { children }\end{array}$ & SWE & 2013 & 1.00 & {$[0.97,1.03]$} & 0.02 & {$[-2.27,2.30]$} & 0.96 \\
\hline
\end{tabular}

disproportional per capita number of specialists. The number of privately insured individuals is also strongly super-linear in Brazil (health care is public and completely free in Brazil) whereas in the USA the elderly is, on average, equally insured independently of the city size. Medicare enrollees (social insurance program for elderly and young people with disabilities in the USA), on the other hand, are more concentrated in smaller cities. In Sweden, visits to physicians and dentists are equally distributed across the cities. In general, we observe that access to different forms of health care services is relatively more abundant in larger cities. These facts may be connected to the relative lower burden of non-communicable diseases, suicides, and child and infant mortality observed in larger cities (Table 2), but stronger causal conclusions depend on more carefully analysis of country-specific data.

The exponent $\alpha=1$ indicates a relative equilibrium between the respective variable and the population size across cities within a country. For example, doubling the population results on two times more problems or benefits. This equilibrium is not 


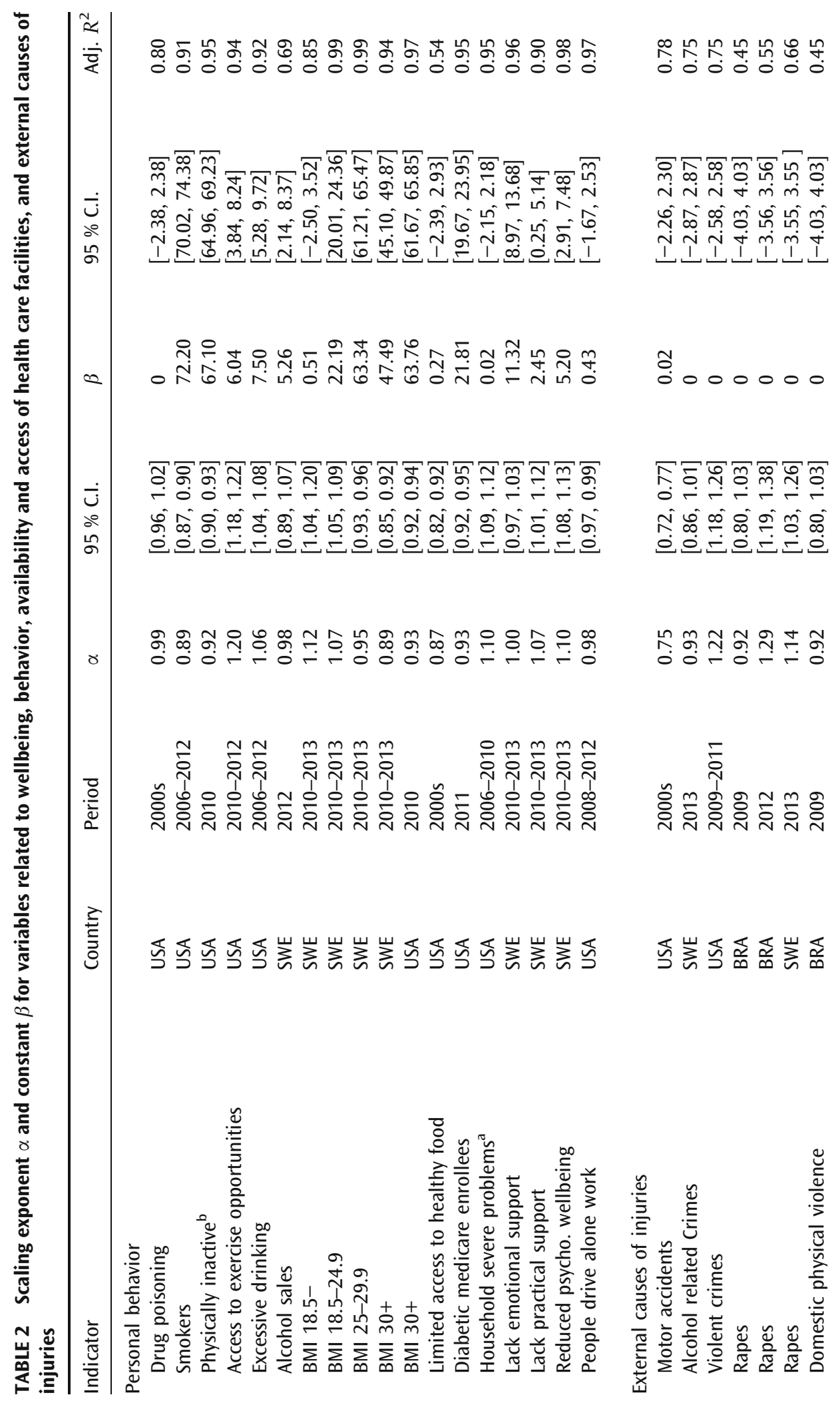




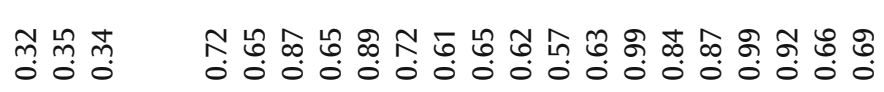







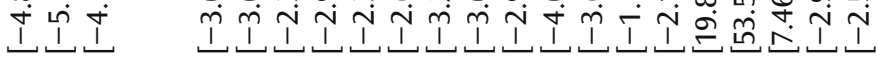

$000 \quad 00000000000000000$



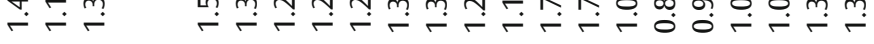
mิ

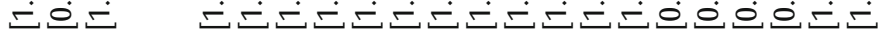

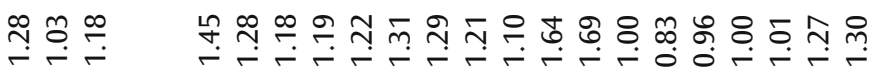

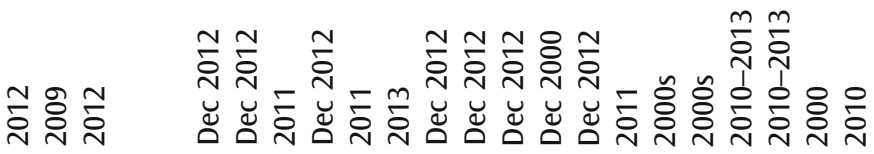

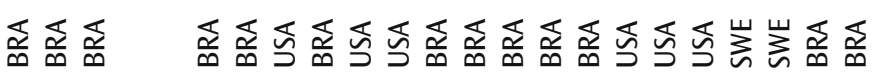

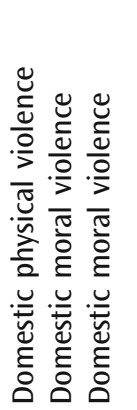

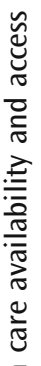



$\frac{\sim}{\frac{0}{0}} \frac{\tilde{0}}{\frac{0}{0}}$





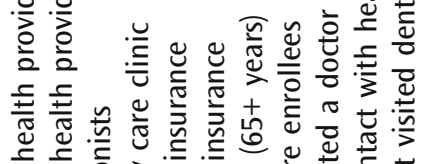

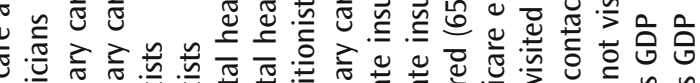

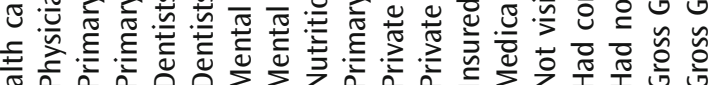
 
necessarily optimal because it may indicate that physical resources may be underexploited, as for example, facilities for physical activity or number of beds in hospitals. While there is no clear stable or optimal point for this exponent, deviations from the linearity suggest that resources should not be proportionally allocated if one wishes to promote a similar quality of life to the entire country.

Efforts to promote equality may thus be reflected in the evolution of the scaling exponents, which are not expected to be constant in time. Considering the Brazilian context, Fig. 2 shows that HIV affected smaller cities more severely in the early stage of the epidemics in the 1980 s. As the epidemics pervaded the population, larger cities have disproportionally suffered. In other words, HIV more easily spread in larger cities. Infant mortality, diabetes, and heart attacks have all approached linear
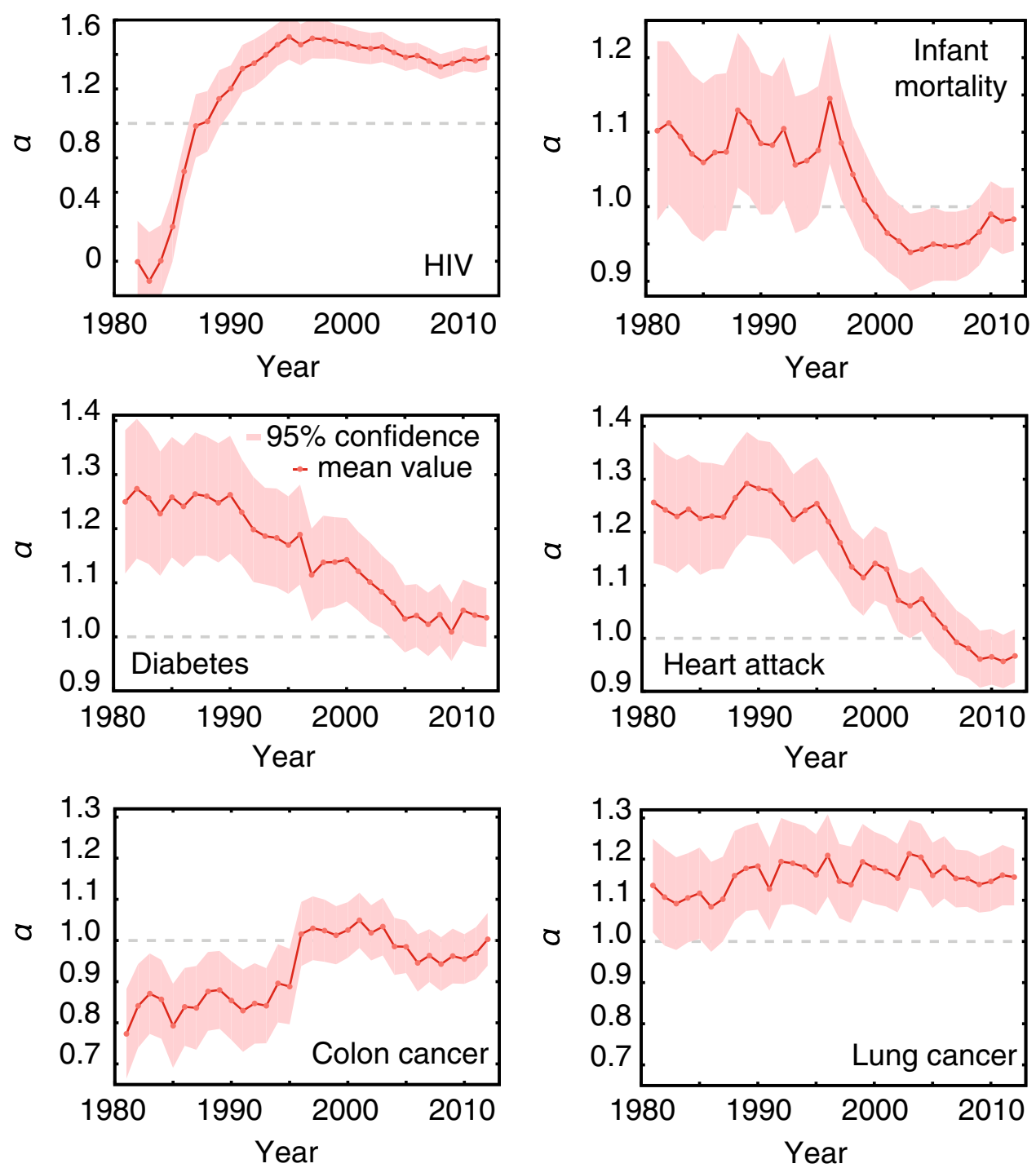

FIG. 2 The panels show the variation of the scaling exponent $\alpha$ (together with the $95 \%$ C.I. (shade area)) for selected variables in the period from 1981 to 2012 in Brazil. Dotted horizontal lines correspond to the reference of linear scaling $\alpha=1$. 
scaling in recent years. For diabetes and heart attacks, it is difficult to infer if the improvement is a result of better health in larger cities or better diagnosis in smaller cities. Deaths by lung cancer have maintained super-linear exponents over the 30year period. On the other hand, deaths by colon cancer have affected relatively more people in larger cities in recent years than in the $1980 \mathrm{~s}$. Convergence to linear or sub-linear exponents in these cases is a positive sign of an increasing urban advantage.

We have been looking at the scaling properties of different cities at a given year. These analyses provide comparative information at the national level but little say regarding the development of a single city. The health variables discussed above are not expected to change equally across cities when the cities increase in size. Each city in fact goes through a different dynamics over time (e.g., investments, education) that affects health in one way or another even in cities with similar size (a phenomenon known as the Glasgow effect ${ }^{36}$ ). If we perform the previous analysis for single cities in Brazil at different years, we observe that the scaling properties vary substantially for some health variables in selected cities of different sizes (Table 3). HIV is a particular case because the epidemics started in the early 1980s, experiencing a substantial growth in the early years in some cities, followed by a slower but still high incidence after the peak of the epidemics in the early 1990s. Infant mortality on the other hand had a negative strong variation in the exponent $\alpha$, a positive sign reflecting a strong decrease in the number of deaths over the years. Only for the city of Belo Horizonte that we observe a sub-linear exponent for diabetes whereas the number of heart attacks varies for each city. The number of cases of colon and lung cancer is in general strongly super-linear with the exception of colon cancer in Campinas $(\alpha=-1.77)$ and Belo Horizonte $(\alpha=0.32)$.

\section{DISCUSSIONS}

By analyzing the scaling relation between the population size of cities and various health-related variables, we find that in general, those conditions directly involving interaction between people scales super-linearly with the population size. This is particularly the case of various infectious diseases, with an exception of those linked to resource-poor environments such as dengue and leprosy. This means that larger cities have a relatively higher incidence of infectious diseases that translates into a relative disadvantage in respect to smaller cities. These results suggest that infectious diseases spread relatively faster within denser agglomeration of people, possibly a result of the non-linear contact patterns between individuals (people make a higher number of per capita contacts in larger cities ${ }^{26}$ ) and the human mobility patterns. ${ }^{37}$ Similarly, the number of violent crimes, rapes, domestic violence, and household problems increases super-linearly with population. On the other hand, the number of deaths by suicides, heart attacks, or cases of diabetes is either sub-linear or close to linear depending on the country studied. This means that the number of deaths by these factors is relatively smaller in larger cities, that is, large city dwellers are relatively less likely to commit suicide, die by a heart attack, or develop diabetes than those living in smaller cities. This could be linked to the relative disproportion of elderly people living in smaller cities, ${ }^{38}$ but strong conclusions require further studies. These results also relate to our findings that obesity (USA and Sweden), smoking (USA), physical inactivity (USA), and limited access to health food (USA) all scale sub-linearly with population size considering 


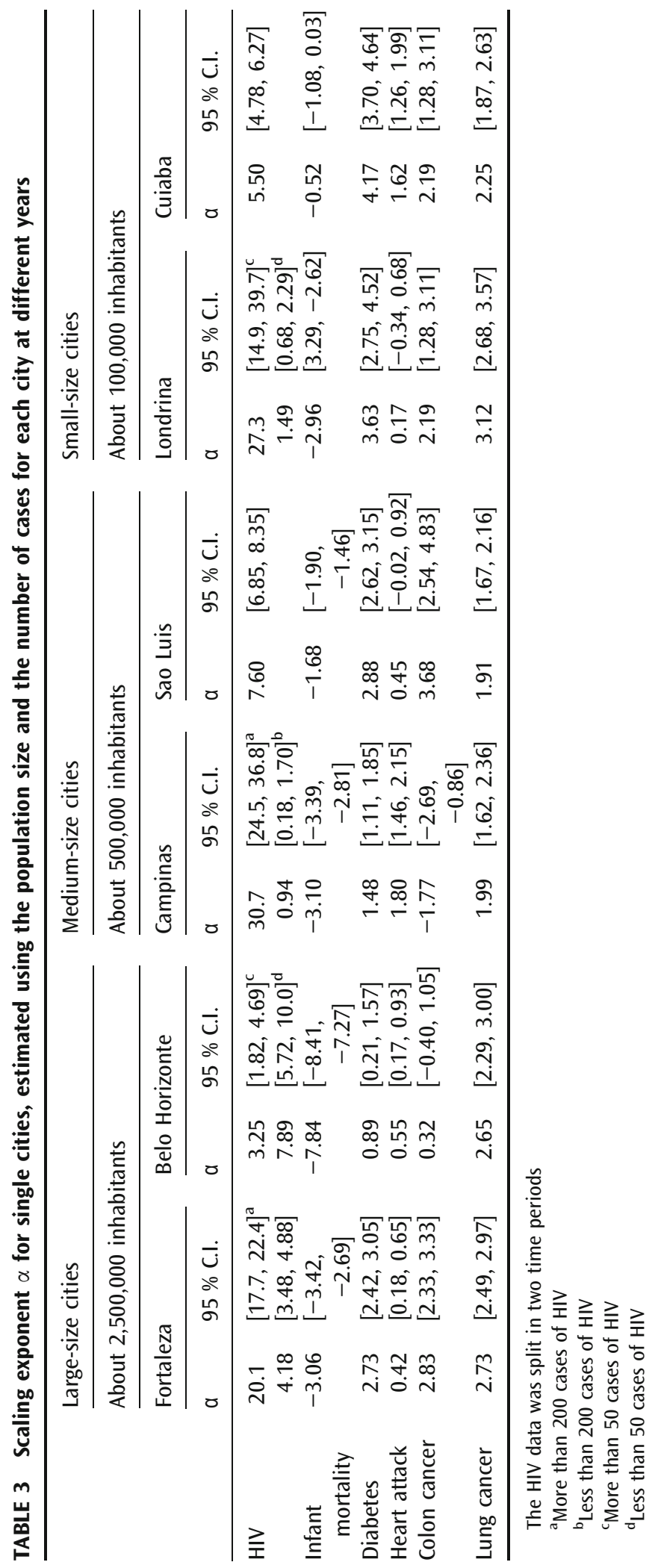


the available data, indicating that relatively speaking dwellers of larger cities have on average a healthier lifestyle, if only those variables are considered, possibly benefiting of the social networks. ${ }^{39}$ Preliminary data in the context of Brazil however indicate that smoking increases super-linearly with population size, suggesting that healthy lifestyle may not be a universal characteristic of larger cities. Furthermore, previous studies found that the incidence of psychosis and depression increases with the population density ${ }^{40,41}$ suggesting that mental health is more sensitive to urban living. Lung cancer and chronic respiratory insufficiency are both relatively more likely in larger cities of Brazil whereas they are relatively less likely in larger cities of Sweden. Lung cancer is linked to smoking that is weakly super-linear in Brazil. Pollution, relatively higher in larger cities, also plays a role on these diseases and may, at least partially, explain these findings. ${ }^{42}$ More robust data for the various countries are necessary before making stronger conclusions, at the macroscopic level, between pollution, smoking, lung cancer, and population size of cities.

Previous empirical studies show that the density of ambulatory hospitals in the USA and primary care clinics in South Korea scales super-linearly with the population density within a certain area. South Korea hospitals on the other hand scale slightly sub-linearly. ${ }^{43}$ These results indicate that primary care facilities have a high availability in densely populated areas. Similarly, we find that in both Brazil and the USA, the availability of specialist and primary care physicians, dentists, mental health providers, and nutritionists all scale strongly super-linearly with the population size. Primary care clinics and people with private insurance also increase super-linearly in Brazil. Altogether, these results show that the availability of health care per capita is significantly higher in larger cities in those countries. Although larger cities are attraction poles of medical services for dwellers of smaller cities, the easier and increasing access to medical facilities in larger cities may explain why the number of deaths by non-external causes is continuously getting less super-linear in Brazil.

The most important limitation of this methodology is that we are unable to make causal relations between population size and health outcomes. We cannot directly use the non-linearity of one quantity to explain the scaling of another. Moreover, while several scaling exponents are similar to different countries, sometimes, they give contrasting results suggesting that intrinsic characteristics of each country, such as public policies, income, cultural aspects, or even the country own geography, may be defining the health outcomes and limiting the universality of the results. Brazil, Sweden, and the USA are relatively different countries yet they share similarities. If data from other countries, particularly those in the low-income bracket, become available, a theory on non-linear scaling of health variables could be further developed. Non-standardized and missing data also restrict a detailed comparison across countries. Recent studies have questioned the definition of city boundaries and thus the non-linear scaling with population size for some urban indicators. ${ }^{13,44,45}$ Altogether, these results however help to understand the effects of the increasing complexity of larger cities in public health. For some diseases, higher per capita efforts should be given to larger cities, whereas for other diseases, efforts should target smaller cities. Another important lesson is that ranks based on simple rates miss the effects of population size and thus provide an incomplete picture of the state of health of individual cities across a country. One should therefore also look at the deviations from linearity in order to more accurately estimate trends and rank cities. 


\section{ACKNOWLEDGMENTS}

LECR is a FNRS chargé de recherches.

\section{REFERENCES}

1. United Nations, Department of Economic and Social Affairs, Population Division. World Urbanization Prospects, the 2011 Revision. United Nations: New York; 2012.

2. United Nations, Department of Economic and Social Affairs, Population Division. World Urbanization Prospects, the 2014 Revision, Highlights (ST/ESA/SER.A/352). United Nations: New York. 2014.

3. Leon DA. Cities, urbanization and health. Int J Epidemiol. 2008; 37: 4-8.

4. Vlahov D, Galea S, Gibble E, Freudenberg N. Perspectives on urban conditions and population health. Cad Saude Publica. 2005; 21: 949-57.

5. National Research Council. Mortality and morbidity. Is city life good for your health? in Cities Transformed. Demographic Change and Its Implications in the Developing World. 7th ed. Washington DC: The National Academies Press; 2003.

6. Buch T, Hamann S, Niebuhr A, Rossen A. What makes cities attractive? The determinants of urban labour migration in Germany. Urban Stud. 2014; 51: 1960-78.

7. Jacobs J. The economy of cities. Vintage Publisher 1970. 288p

8. Bettencourt LMA, Lobo J, Helbing D, Kühnert C, West GB. Growth, innovation, scaling, and the pace of life in cities. PNAS. 2007; 104: 7301-6.

9. Bettencourt LMA, Lobo J, Strumsky D, West GB. Urban scaling and its deviations. Revealing the structure of wealth, innovation and crime across cities. PLOS ONE. 2010; 5: e13541.

10. Gomez-Lievano A, Youn H, Bettencourt LMA. The statistics of urban scaling and their connection to Zipf's law. PLoS ONE. 2012; 7: e40393.

11. Sarzynski A. Bigger is not always better. A comparative analysis of cities and their air pollution impact. Urban Stud. 2012; 49: 3121-38.

12. Fragkias M, Lobo J, Strumsky D, Seto KC. Does size matter? Scaling of CO2 emissions and U.S. urban areas. PLoS ONE. 2013; 8: e64727.

13. Oliveira EA, Andrade JS Jr, Makse HA. Large cities are less green. Sci Rep. 2014; 5(4235).

14. Peen J, Schoevers RA, Beekman AT, Dekker J. The current status of urban-rural differences in psychiatric disorders. Acta Psychiatr Scand. 2010; 121: 84-93.

15. Rydin Y, Bleahu A, Davies M, et al. Shaping cities for health: complexity and the planning of urban environments in the 21st century. Lancet. 2012; 379: 2079-108.

16. Cyril S, Oldroyd JC, Renzaho A. Urbanisation, urbanicity, and health. A systematic review of the reliability and validity of urbanicity scales. BMC Public Health. 2013; 13(513).

17. Eve RA, Horsfall ST, Lee M. Chaos, complexity, and sociology. Myths, models, and theories. SAGE Publications, Inc. Thousand Oaks: California; 1997. 360p.

18. Mitchell M. Complexity. A guided tour. New York, NY: Oxford University Press; 2011. 368p.

19. Shingleton A. Allometry: the study of biological scaling. Nat Educ Know. 2010; 3(2).

20. West GB, Brown JH, Enquist BJ. A general model for the origin of allometric scaling laws in biology. Science. 1997; 276: 122-6.

21. Martinez J, Pampalon R, Hamel D, Raymond G. Does living in rural communities rather than cities really make a difference in people's health and wellness? Institut national de santé publique du Quebec 2004

22. Galea S, Freudenberg N, Vlahov D. Cities and population health. Soc Sci Med. 2005; 60: 1017-33.

23. Melo HPM, Moreira AA, Batista E, Makse HA, Andrade JS. Statistical signs of social influence on suicides. Sci Rep. 2014; 4(6239). 
24. Antonio FJ, De Picoli S Jr, Teixeira JJV, Mendes RS. Growth patterns and scaling laws governing aids epidemic in Brazilian cities. PLoS ONE. 2014; 9: e111015.

25. Anderson RM, May RM. Infectious diseases of humans. Dynamics and control. New York, NY: Oxford University Press; 1991. 768p.

26. Schlapfer M, Bettencourt LMA, Grauwin S, et al. The scaling of human interactions with city size. J R Soc Interface. 2014; 11(20130789).

27. Acuna-Soto R, Viboud C, Chowell G. Influenza and pneumonia mortality in 66 large cities in the United States in years surrounding the 1918 pandemic. PLOS ONE. 2011; 6: e23467.

28. Britton WJ, Lockwood DN. Leprosy. Lancet. 2004; 363: 1209-19.

29. Gubler DJ. The economic burden of dengue. Am J Trop Med Hyg. 2012; 86: 743-4.

30. Malta DC, Iser BPM, de Sá NNB, et al. Tendências temporais no consumo de tabaco nas capitais brasileiras, segundo dados do VIGITEL 2006, a 2011. Cad Saúde Pública. 2013; 29: 812-22.

31. Christakis NA, Fowler JH. The spread of obesity in a large social network over 32 years. N Engl J Med. 2007; 357: 370-9.

32. Gallos LK, Barttfeld P, Havlin S, Sigman M, Makse HA. Collective behavior in the spatial spreading of obesity. Sci Rep. 2012; 2(454).

33. Christakis NA, Fowler JH. The collective dynamics of smoking in a large social network. N Engl J Med. 2008; 358: 2249-58.

34. Lobo J, Bettencourt LMA, Strumsky D, West GB. Urban scaling and the production function for cities. PLoS ONE. 2013; 8: e58407.

35. Sornette D, Maillart T, Ghezzi G. How much is the whole really more than the sum of its parts? $1+1=2.5$. Superlinear productivity in collective group actions. PLoS ONE. 2014; 9: e103023.

36. Walsh D, Bendel N, Jones R, Hanlon P. It's not 'just deprivation': why do equally deprived UK cities experience different health outcomes? Public Health. 2010; 124: 48795.

37. Dalziel BD, Pourbohloul B, Ellner SP. Human mobility patterns predict divergent epidemic dynamics among cities. Proc R Soc B. 2013; 280: 1766.

38. Jahan NK, Allotey P, Arunachalam D, et al. The rural bite in population pyramids. What are the implications for responsiveness of health systems in middle income countries? BMC Public Health. 2014; 14(2): S8.

39. O'Malley AJ, Arbesman S, Steiger DM, Fowler JH, Christakis NA. Egocentric social network structure, health, and pro-social behaviors in a national panel study of Americans. PLoS One. 2012; 7: e36250.

40. Sundquist K, Frank G, Sundquist J. Urbanisation and incidence of psychosis and depression. Follow-up study of 4.4 million women and men in Sweden. Br J Psychiatry. 2004; 184: 293-8.

41. Vigod SN, Tarasoff LA, Bryja B, et al. Relation between place of residence and postpartum depression. CMAJ. 2013; 185: 1129-35.

42. Afroz R, Hassan MN, Ibrahim NA. Review of air pollution and health impacts in Malaysia. Environ Res. 2003; 92: 71-7.

43. Um J, Son S-W, Lee S-I, Jeong H, Kim BJ. Scaling laws between population and facility densities. PNAS. 2009; 106: 14236-40.

44. Rozenfeld HD, Rybski D, Gabaix X, Makse HA. The area and population of cities: new insights from a different perspective on cities. Am Econ Rev. 2011; 101: 2205-25.

45. Arcaute E, Hatna E, Ferguson P, et al. Constructing cities, deconstructing scaling laws. $J$ R Soc Interface. 2014; 12: 20140745. 